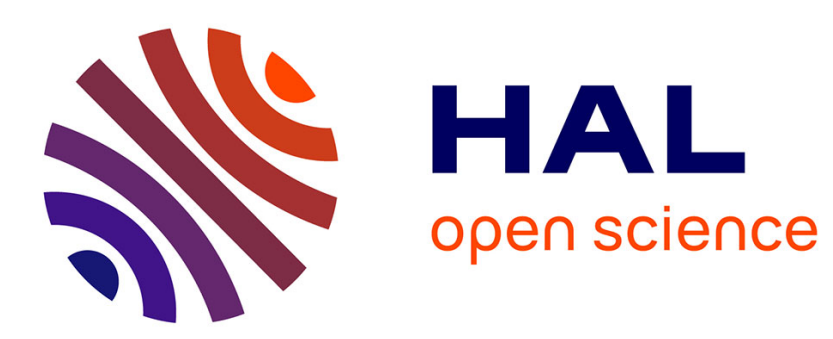

\title{
Sensitivity Comparison of Planar Parallel Manipulators
}

Nicolas Binaud, Stéphane Caro, Philippe Wenger

\section{To cite this version:}

Nicolas Binaud, Stéphane Caro, Philippe Wenger. Sensitivity Comparison of Planar Parallel Manipulators. Mechanism and Machine Theory, 2009, 45, pp.1477-1490. 10.1016/j.mechmachtheory.2010.07.004 . hal-00833527

\section{HAL Id: hal-00833527 \\ https://hal.science/hal-00833527}

Submitted on 13 Jun 2013

HAL is a multi-disciplinary open access archive for the deposit and dissemination of scientific research documents, whether they are published or not. The documents may come from teaching and research institutions in France or abroad, or from public or private research centers.
L'archive ouverte pluridisciplinaire HAL, est destinée au dépôt et à la diffusion de documents scientifiques de niveau recherche, publiés ou non, émanant des établissements d'enseignement et de recherche français ou étrangers, des laboratoires publics ou privés. 


\title{
Sensitivity Comparison of Planar Parallel Manipulators
}

\author{
Nicolas Binaud Stéphane Caro* Philippe Wenger \\ Institut de Recherche en Communications et Cybernétique de Nantes \\ UMR CNRS n 6597 \\ 1 rue de la Noë, 44321 Nantes, France \\ \{nicolas.binaud, stephane.caro, philippe.wenger\}@irccyn.ec-nantes.fr
}

\begin{abstract}
This paper deals with the sensitivity comparison of three Degree-of-Freedom planar parallel manipulators. First, a methodology is described to obtain the sensitivity coefficients of the pose of the moving platform of the manipulators to variations in their geometric parameters and actuated variables. Their sensitivity coefficients are derived and expressed algebraically for a matter of analysis simplicity. Moreover, two aggregate sensitivity indices are determined, the first one is related to the orientation of the moving platform of the manipulator and the other one to its position. Then, a methodology is proposed to compare PPMs with regard to

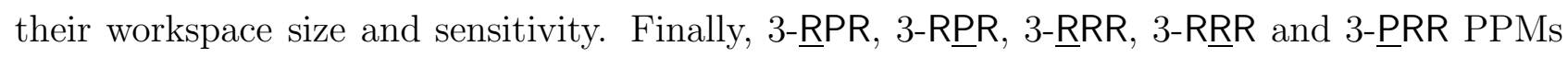
are compared in order to highlight the contributions of the paper.
\end{abstract}

\section{Introduction}

During the early design process of engineering systems, the analysis of the performance sensitivity to uncertainties is an important task. High sensitivity to parameters that are inherently noisy can lead to poor, or unexpected performance. In robotics, the variations in the geometric parameters of Parallel Kinematics Machines (PKMs) can be either compensated or amplified. For that reason, it is important to analyze the sensitivity of their performance to variations in its geometric parameters. Accordingly, it turns to be very useful to develop a methodology for the comparison of the sensitivity of PKMs to uncertainties at their conceptual design stage. Ideally, having this information at the conceptual design stage can help robot designers better choose the architecture of the manipulator under design. To this end, some indices such as the dexterity and the manipulability have been used to evaluate the sensitivity of robots performance to variations in their actuated joints [1, 2, 3, 4, 5]. However, they are not suitable for

${ }^{*}$ Corresponding author: Stéphane Caro; Email: stephane.caro@irccyn.ec-nantes.fr; Tel: +33 24037 69 68; Fax: +33240376930 
the evaluation of this sensitivity to other types of uncertainty such as variations in geometric parameters.

Two indices are proposed in [6] to evaluate the sensitivity of the end-effector pose (position + orientation) of the Orthoglide 3-axis, a three Degree-of-Freedom (DOF) translational PKM, to variations in its design parameters. In the same vein, four 3-RPR planar parallel manipulators (PPMs) are compared in [7] based on the sensitivity of their performance to variations in their geometric parameters. However, as far as the authors know, there is no work in the literature related to such a comparison of manipulators with different architectures. Therefore, this paper introduces a methodology to compare different types of PPMs based on the sensitivity of their moving platform pose to variations in their geometric parameters. Only manipulators with the same architecture were compared in [7] and [9], whereas manipulators of different architectures are compared in this paper, namely, the 3-RPR, the 3-RRR and the 3-PRR PPMs. The architectures of the manipulators under study are first described. Then, the sensitivity coefficients of the pose of their moving platform to variations in their geometric parameters and actuated variables are derived and expressed algebraically. Moreover, two aggregate sensitivity indices are determined, one is related to the orientation of the moving platform of the manipulator and another one is related to its position. Then, a methodology is proposed to compare PPMs

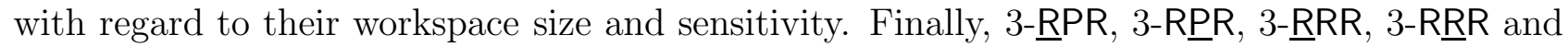
3-PRR PPMs are compared as illustrative examples.

\section{Manipulators Architecture}

Here and throughout this paper, $\mathrm{R}, \mathrm{P}, \underline{\mathrm{R}}$ and $\underline{\mathrm{P}}$ denote revolute, prismatic, actuated revolute and actuated prismatic joints, respectively. Figures 1(a)-(c) illustrate the architectures of the manipulators under study, namely the 3-RPR, the 3-RRR and the the 3-PRR PPMs, respectively. They are composed of a base and a moving platform (MP) connected by means of three legs. Points $A_{1}, A_{2}$ and $A_{3},\left(C_{1}, C_{2}\right.$ and $C_{3}$, respectively) lie at the corners of a triangle, point $O$ (point $P$, resp.) being its circumcenter. Each leg is composed of three joints in sequence, one of them being actuated. For example, each leg of the 3-RRR PPM is composed of three revolute joints mounted in sequence, the first one being actuated.

$\mathcal{F}_{b}$ and $\mathcal{F}_{p}$ are the base and the moving platform frames of the manipulator. In the scope of this paper, both $\mathcal{F}_{b}$ and $\mathcal{F}_{p}$ are supposed to be orthogonal. $\mathcal{F}_{b}$ is defined with the orthogonal dihedron $(\overrightarrow{O x}, \overrightarrow{O y})$, point $O$ is its center and $\overrightarrow{O x}$ is parallel to segment $A_{1} A_{2}$. Likewise, $\mathcal{F}_{p}$ is defined with the orthogonal dihedron $(\overrightarrow{P X}, \overrightarrow{P Y})$, point $P$ is its center and $\overrightarrow{P X}$ is parallel to segment $C_{1} C_{2}$.

The MP pose, i.e., its position and its orientation, is determined by means of the Cartesian coordinates vector $\mathbf{p}=\left[p_{x}, p_{y}\right]^{T}$ of operation point $P$ expressed in $\mathcal{F}_{b}$ and angle $\phi$, that is the rotation angle between frames $\mathcal{F}_{b}$ and $\mathcal{F}_{p}$. 


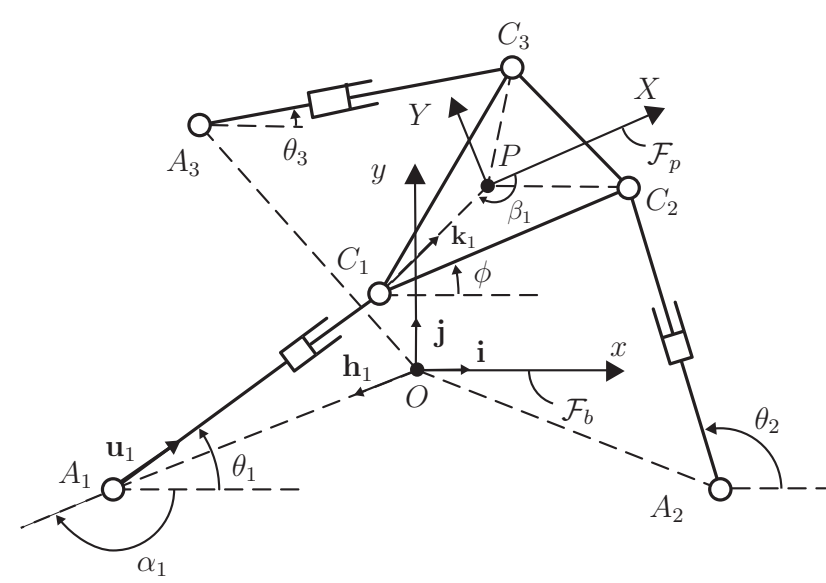

(a) 3-RPR PPM

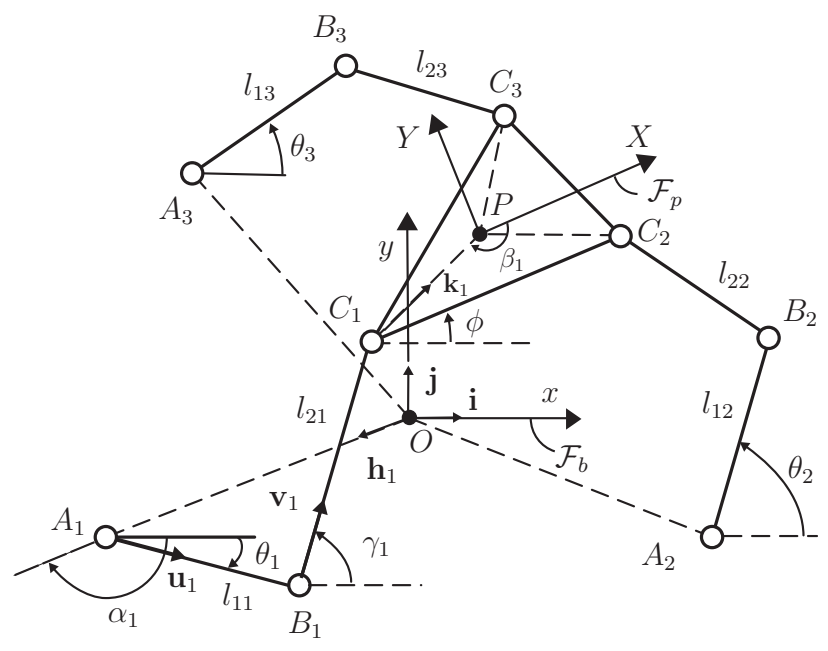

(b) 3-RRR PPM

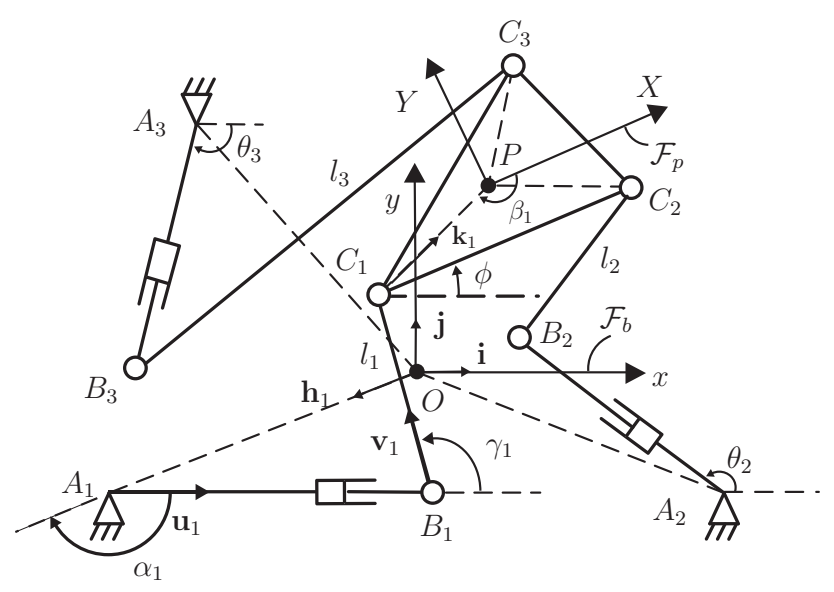

(c) 3-PRR PPM

Figure 1: PPMs under study

\section{Sensitivity Indices}

In this section, we first introduce a methodology to derive the sensitivity coefficients of the MP pose of the PPMs to variations in the actuated joints, in the leg lengths as well as in the coordinates of points $A_{i}$ and $C_{i}, i=1,2,3$, the latter being either Polar or Cartesian. In [7] and [9], this methodology was illustrated with a $3-R \underline{P R}$ and a $3-\underline{R} R R$ PPMs, respectively. Here, it is illustrated with a 3 -PRR PPM. From the foregoing sensitivity coefficients, we propose two aggregate sensitivity indices, one related to the position of the MP and another one related to its orientation.

\subsection{Sensitivity Coefficients}

We focus on the 3-PRR PPM to illustrate the methodology used to derive the sensitivity coefficients of any PPM. From the closed-loop kinematic chains $O-A_{i}-B_{i}-C_{i}-P-O, i=1, \ldots, 3$ 
depicted in Fig. 1(c), the position vector $\mathbf{p}$ of point $P$ can be expressed in $\mathcal{F}_{b}$ as follows,

$$
\mathbf{p}=\left[\begin{array}{c}
p_{x} \\
p_{y}
\end{array}\right]=\mathbf{a}_{i}+\left(\mathbf{b}_{i}-\mathbf{a}_{i}\right)+\left(\mathbf{c}_{i}-\mathbf{b}_{i}\right)+\left(\mathbf{p}-\mathbf{c}_{i}\right), \quad i=1, \ldots, 3
$$

$\mathbf{a}_{i}, \mathbf{b}_{i}$ and $\mathbf{c}_{i}$ being the position vectors of points $A_{i}, B_{i}$ and $C_{i}$ expressed in $\mathcal{F}_{b}$. Equation (??) can also be written as,

$$
\mathbf{p}=a_{i} \mathbf{h}_{i}+\rho_{i} \mathbf{u}_{i}+l_{i} \mathbf{v}_{i}+c_{i} \mathbf{k}_{i}
$$

with

$$
\mathbf{h}_{i}=\left[\begin{array}{c}
\cos \alpha_{i} \\
\sin \alpha_{i}
\end{array}\right], \mathbf{u}_{i}=\left[\begin{array}{c}
\cos \theta_{i} \\
\sin \theta_{i}
\end{array}\right], \mathbf{v}_{i}=\left[\begin{array}{c}
\cos \gamma_{i} \\
\sin \gamma_{i}
\end{array}\right], \mathbf{k}_{i}=\left[\begin{array}{c}
\cos \left(\phi+\beta_{i}+\pi\right) \\
\sin \left(\phi+\beta_{i}+\pi\right)
\end{array}\right]
$$

where $a_{i}$ is the distance between points $O$ and $A_{i}, \rho_{i}$ is the distance between points $A_{i}$ and $B_{i}, l_{i}$ is the distance between points $B_{i}$ and $C_{i}, c_{i}$ is the distance between points $C_{i}$ and $P$, $\mathbf{h}_{i}$ is the unit vector $\overrightarrow{O A_{i}} /\left\|\overrightarrow{O A_{i}}\right\|_{2}, \mathbf{u}_{i}$ is the unit vector $\overrightarrow{A_{i} B_{i}} /\left\|\overrightarrow{A_{i} B_{i}}\right\|_{2}, \mathbf{v}_{i}$ is the unit vector $\overrightarrow{B_{i} C_{i}} /\left\|\overrightarrow{B_{i} C_{i}}\right\|_{2}$ and $\mathbf{k}_{i}$ is the unit vector $\overrightarrow{C_{i} P} /\left\|\overrightarrow{C_{i} P}\right\|_{2}$.

In a manner similar to [7, 8], upon differentiation of Eq.(??), we obtain:

$$
\begin{aligned}
\delta \mathbf{p}= & \delta a_{i} \mathbf{h}_{i}+a_{i} \delta \alpha_{i} \mathbf{E} \mathbf{h}_{i}+\delta \rho_{i} \mathbf{u}_{i}+\rho_{i} \delta \theta_{i} \mathbf{E} \mathbf{u}_{i}+\delta l_{i} \mathbf{v}_{i}+l_{i} \delta \gamma_{i} \mathbf{E} \mathbf{v}_{i}+\delta c_{i} \mathbf{k}_{i} \\
& +c_{i}\left(\delta \phi+\delta \beta_{i}\right) \mathbf{E} \mathbf{k}_{i}
\end{aligned}
$$

with matrix $\mathbf{E}$ defined as

$$
\mathbf{E}=\left[\begin{array}{cc}
0 & -1 \\
1 & 0
\end{array}\right]
$$

$\delta \mathbf{p}$ and $\delta \phi$ being the position and orientation errors of the MP. Likewise, $\delta a_{i}, \delta \alpha_{i}, \delta \rho_{i}, \delta l_{i}, \delta c_{i}$ and $\delta \beta_{i}$ denote the variations in $a_{i}, \alpha_{i}, \rho_{i}, l_{i}, c_{i}$ and $\beta_{i}$, respectively. The idle variation $\delta \gamma_{i}$ is eliminated by dot-multiplying Eq.(??) with $l_{i} \mathbf{v}_{i}^{T}$, thus obtaining

$$
\begin{aligned}
l_{i} \mathbf{v}_{i}^{T} \delta \mathbf{p}= & l_{i} \delta a_{i} \mathbf{v}_{i}^{T} \mathbf{h}_{i}+l_{i} a_{i} \delta \alpha_{i} \mathbf{v}_{i}^{T} \mathbf{E h}_{i}+l_{i} \delta \rho_{i} \mathbf{v}_{i}^{T} \mathbf{u}_{i}+l_{i} \rho_{i} \delta \theta_{i} \mathbf{v}_{i}^{T} \mathbf{E} \mathbf{u}_{i}+l_{i} \delta l_{i} \\
& +l_{i} \delta c_{i} \mathbf{v}_{i}^{T} \mathbf{k}_{i}+l_{i} c_{i}\left(\delta \phi+\delta \beta_{i}\right) \mathbf{v}_{i}^{T} \mathbf{E k}_{i}
\end{aligned}
$$

Equation (??) can now be cast in vector form:

$$
\begin{aligned}
\mathbf{A}\left[\begin{array}{c}
\delta \phi \\
\delta \mathbf{p}
\end{array}\right]= & \mathbf{H}_{a}\left[\begin{array}{c}
\delta a_{1} \\
\delta a_{2} \\
\delta a_{3}
\end{array}\right]+\mathbf{H}_{\alpha}\left[\begin{array}{l}
\delta \alpha_{1} \\
\delta \alpha_{2} \\
\delta \alpha_{3}
\end{array}\right]+\mathbf{B}\left[\begin{array}{c}
\delta \rho_{1} \\
\delta \rho_{2} \\
\delta \rho_{3}
\end{array}\right]+\mathbf{H}_{\theta}\left[\begin{array}{c}
\delta \theta_{1} \\
\delta \theta_{2} \\
\delta \theta_{3}
\end{array}\right]+\mathbf{H}_{l}\left[\begin{array}{l}
\delta l_{1} \\
\delta l_{2} \\
\delta l_{3}
\end{array}\right] \\
& +\mathbf{H}_{c}\left[\begin{array}{c}
\delta c_{1} \\
\delta c_{2} \\
\delta c_{3}
\end{array}\right]+\mathbf{H}_{\beta}\left[\begin{array}{c}
\delta \beta_{1} \\
\delta \beta_{2} \\
\delta \beta_{3}
\end{array}\right]
\end{aligned}
$$


with

$$
\begin{aligned}
& \mathbf{A}=\left[\begin{array}{ll}
m_{1} & l_{1} \mathbf{v}_{1}^{T} \\
m_{2} & l_{2} \mathbf{v}_{2}^{T} \\
m_{3} & l_{3} \mathbf{v}_{3}^{T}
\end{array}\right], \mathbf{B}=\operatorname{diag}\left[\begin{array}{lll}
l_{1} \mathbf{v}_{1}^{T} \mathbf{u}_{1} & l_{2} \mathbf{v}_{2}^{T} \mathbf{u}_{2} & l_{3} \mathbf{v}_{3}^{T} \mathbf{u}_{3}
\end{array}\right] \\
& \mathbf{H}_{a}=\operatorname{diag}\left[\begin{array}{llll}
l_{1} \mathbf{v}_{1}^{T} \mathbf{h}_{1} & l_{2} \mathbf{v}_{2}^{T} \mathbf{h}_{2} & l_{3} \mathbf{v}_{3}^{T} \mathbf{h}_{3}
\end{array}\right] \\
& \mathbf{H}_{\alpha}=\operatorname{diag}\left[\begin{array}{lll}
l_{1} a_{1} \mathbf{v}_{1}^{T} \mathbf{E} \mathbf{h}_{1} & l_{2} a_{2} \mathbf{v}_{2}^{T} \mathbf{E h}_{2} & l_{3} a_{3} \mathbf{v}_{3}^{T} \mathbf{E h}_{3}
\end{array}\right] \\
& \mathbf{H}_{l}=\operatorname{diag}\left[\begin{array}{lll}
l_{1} & l_{2} & l_{3}
\end{array}\right] \\
& \mathbf{H}_{\theta}=\operatorname{diag}\left[\begin{array}{lll}
l_{1} \rho_{1} \mathbf{v}_{1}^{T} \mathbf{E} \mathbf{h}_{1} & l_{2} \rho_{2} \mathbf{v}_{2}^{T} \mathbf{E h}_{2} & l_{3} \rho_{3} \mathbf{v}_{3}^{T} \mathbf{E h}_{3}
\end{array}\right] \\
& \mathbf{H}_{c}=\operatorname{diag}\left[\begin{array}{lll}
l_{1} \mathbf{v}_{1}^{T} \mathbf{k}_{1} & l_{2} \mathbf{v}_{2}^{T} \mathbf{k}_{2} & l_{3} \mathbf{v}_{3}^{T} \mathbf{k}_{3}
\end{array}\right] \\
& \mathbf{H}_{\beta}=\operatorname{diag}\left[\begin{array}{lll}
l_{1} c_{1} \mathbf{v}_{1}^{T} \mathbf{E} \mathbf{k}_{1} & l_{2} c_{2} \mathbf{v}_{2}^{T} \mathbf{E} \mathbf{k}_{2} & l_{3} c_{3} \mathbf{v}_{3}^{T} \mathbf{E} \mathbf{k}_{3}
\end{array}\right]
\end{aligned}
$$

and

$$
m_{i}=-l_{i} c_{i} \mathbf{v}_{i}^{T} \mathbf{E k}_{i}, \quad i=1, \ldots, 3
$$

Let us notice that $\mathbf{A}$ and $\mathbf{B}$ are the direct and the inverse Jacobian matrices of the manipulator, respectively. Assuming that $\mathbf{A}$ is non singular, i.e., the manipulator does not meet any Type II singularity [10, 11, 12, 13], we obtain upon multiplication of Eq.(??) by $\mathbf{A}^{-1}$ :

$$
\begin{aligned}
{\left[\begin{array}{l}
\delta \phi \\
\delta \mathbf{p}
\end{array}\right]=} & \mathbf{J}_{a}\left[\begin{array}{l}
\delta a_{1} \\
\delta a_{2} \\
\delta a_{3}
\end{array}\right]+\mathbf{J}_{\alpha}\left[\begin{array}{l}
\delta \alpha_{1} \\
\delta \alpha_{2} \\
\delta \alpha_{3}
\end{array}\right]+\mathbf{J}\left[\begin{array}{l}
\delta \rho_{1} \\
\delta \rho_{2} \\
\delta \rho_{3}
\end{array}\right]+\mathbf{J}_{\theta}\left[\begin{array}{c}
\delta \theta_{1} \\
\delta \theta_{2} \\
\delta \theta_{3}
\end{array}\right]+\mathbf{J}_{l}\left[\begin{array}{l}
\delta l_{1} \\
\delta l_{2} \\
\delta l_{3}
\end{array}\right] \\
& +\mathbf{J}_{c}\left[\begin{array}{c}
\delta c_{1} \\
\delta c_{2} \\
\delta c_{3}
\end{array}\right]+\mathbf{J}_{\beta}\left[\begin{array}{c}
\delta \beta_{1} \\
\delta \beta_{2} \\
\delta \beta_{3}
\end{array}\right]
\end{aligned}
$$

with

$$
\begin{aligned}
\mathbf{J} & =\mathbf{A}^{-1} \mathbf{B} \\
\mathbf{J}_{a} & =\mathbf{A}^{-1} \mathbf{H}_{a} \\
\mathbf{J}_{\alpha} & =\mathbf{A}^{-1} \mathbf{H}_{\alpha} \\
\mathbf{J}_{\theta} & =\mathbf{A}^{-1} \mathbf{H}_{\theta} \\
\mathbf{J}_{l} & =\mathbf{A}^{-1} \mathbf{H}_{l} \\
\mathbf{J}_{c} & =\mathbf{A}^{-1} \mathbf{H}_{c} \\
\mathbf{J}_{\beta} & =\mathbf{A}^{-1} \mathbf{H}_{\beta}
\end{aligned}
$$

and

$$
\mathbf{A}^{-1}=\frac{1}{\operatorname{det}(\mathbf{A})}\left[\begin{array}{ccc}
v_{1} & v_{2} & v_{3} \\
\mathbf{w}_{1} & \mathbf{w}_{2} & \mathbf{w}_{3}
\end{array}\right]
$$




$$
\begin{aligned}
v_{i} & =l_{j} l_{k}\left(\mathbf{v}_{j} \times \mathbf{v}_{k}\right)^{T} \mathbf{k} \\
\mathbf{w}_{i} & =\mathbf{E}\left(m_{j} l_{k} \mathbf{v}_{k}-m_{k} l_{j} \mathbf{v}_{j}\right) \\
\operatorname{det}(\mathbf{A}) & =\sum_{i=1}^{3} m_{i} v_{i} \\
\mathbf{k} & =\mathbf{i} \times \mathbf{j}
\end{aligned}
$$

$j=(i+1)$ modulo $3 ; k=(i+2)$ modulo $3 ; i=1,2,3$. $\mathbf{J}$ is the kinematic Jacobian matrix of the manipulator whereas $\mathbf{J}_{a}, \mathbf{J}_{\alpha}, \mathbf{J}_{\theta}, \mathbf{J}_{l}, \mathbf{J}_{c}$ and $\mathbf{J}_{\beta}$ are named sensitivity Jacobian matrices of the pose of the MP to variations in $a_{i}, \alpha_{i}, \theta_{i}, l_{i}, c_{i}$ and $\beta_{i}$, respectively. Indeed, the terms of $\mathbf{J}_{a}$, $\mathbf{J}_{\alpha}, \mathbf{J}_{\theta}, \mathbf{J}_{l}, \mathbf{J}_{c}$ and $\mathbf{J}_{\beta}$ are the sensitivity coefficients of the position and the orientation of the MP of the manipulator to variations in length $l_{i}$ and the Polar coordinates of points $A_{i}, B_{i}$ and $C_{i}$. Likewise, $\mathbf{J}$ contains the sensitivity coefficients of the pose of the MP of the manipulator to variations in the prismatic actuated joints. It is noteworthy that all these sensitivity coefficients are expressed algebraically for a matter of simplicity analysis and compactness.

Let $\delta a_{i x}$ and $\delta a_{i y}\left(\delta b_{i x}\right.$ and $\delta b_{i y}$, resp.) denote the position errors of points $A_{i}$ (points $B_{i}$, resp.), $i=1,2,3$, along $\overrightarrow{O x}$ and $\overrightarrow{O y}$, namely, the variations in the Cartesian coordinates of points $A_{i}$ and $B_{i}$. Likewise, let $\delta c_{i X}$ and $\delta c_{i Y}$ denote the position errors of the Cartesian coordinates of points $C_{i}$ along $\overrightarrow{P X}$ and $\overrightarrow{P Y}$. From Fig. 1(c),

$$
\begin{aligned}
& {\left[\begin{array}{l}
\delta a_{i x} \\
\delta a_{i y}
\end{array}\right]=\left[\begin{array}{cc}
\cos \alpha_{i} & -a_{i} \sin \alpha_{i} \\
\sin \alpha_{i} & a_{i} \cos \alpha_{i}
\end{array}\right]\left[\begin{array}{l}
\delta a_{i} \\
\delta \alpha_{i}
\end{array}\right]} \\
& {\left[\begin{array}{l}
\delta b_{i x} \\
\delta b_{i y}
\end{array}\right]=\left[\begin{array}{cc}
0 & -\rho_{i} \sin \theta_{i} \\
0 & \rho_{i} \cos \theta_{i}
\end{array}\right]\left[\begin{array}{c}
\delta \rho_{i} \\
\delta \theta_{i}
\end{array}\right]} \\
& {\left[\begin{array}{l}
\delta c_{i X} \\
\delta c_{i Y}
\end{array}\right]=\left[\begin{array}{cc}
\cos \beta_{i} & -c_{i} \sin \beta_{i} \\
\sin \beta_{i} & c_{i} \cos \beta_{i}
\end{array}\right]\left[\begin{array}{l}
\delta c_{i} \\
\delta \beta_{i}
\end{array}\right]}
\end{aligned}
$$

From Eq.(??), we can notice that variations in the Cartesian coordinates of point $B_{i}$ do not depend on variations in the actuated prismatic joints, $\delta \rho_{i}$, because the influence of variations in geometric parameters and the influence of variations in the actuated joints on the pose of the PPM are analyzed separately. Accordingly, from Eq.(??) and Eqs.(??)-(c), we obtain the following relation between the MP pose error and the variations in the Cartesian coordinates of points $A_{i}, B_{i}$ and $C_{i}$ :

$$
\left[\begin{array}{c}
\delta \phi \\
\delta \mathbf{p}
\end{array}\right]=\mathbf{J}_{A}\left[\begin{array}{l}
\delta a_{1 x} \\
\delta a_{1 y} \\
\delta a_{2 x} \\
\delta a_{2 y} \\
\delta a_{3 x} \\
\delta a_{3 y}
\end{array}\right]+\mathbf{J}\left[\begin{array}{l}
\delta \rho_{1} \\
\delta \rho_{2} \\
\delta \rho_{3}
\end{array}\right]+\mathbf{J}_{B}\left[\begin{array}{c}
\delta b_{1 X} \\
\delta b_{1 Y} \\
\delta b_{2 X} \\
\delta b_{2 Y} \\
\delta b_{3 X} \\
\delta b_{3 Y}
\end{array}\right]+\mathbf{J}_{l}\left[\begin{array}{c}
\delta l_{1} \\
\delta l_{2} \\
\delta l_{3}
\end{array}\right]+\mathbf{J}_{C}\left[\begin{array}{c}
\delta c_{1 X} \\
\delta c_{1 Y} \\
\delta c_{2 X} \\
\delta c_{2 Y} \\
\delta c_{3 X} \\
\delta c_{3 Y}
\end{array}\right]
$$

$\mathbf{J}_{A}, \mathbf{J}_{B}, \mathbf{J}_{C}$ and $\mathbf{J}_{l}$ are named sensitivity Jacobian matrices of the MP pose to variations in $l_{i}$ 
and the Cartesian coordinates of points $A_{i}, B_{i}$, and $C_{i}$, respectively.

In order to better highlight the sensitivity coefficients, let us write the $3 \times 6$ matrices $\mathbf{J}_{A}$, $\mathbf{J}_{B}$ and $\mathbf{J}_{C}$ and the $3 \times 3$ matrices $\mathbf{J}$ and $\mathbf{J}_{l}$ as follows,

$$
\begin{aligned}
\mathbf{J}_{A} & =\left[\begin{array}{lll}
\mathbf{J}_{A_{1}} & \mathbf{J}_{A_{2}} & \mathbf{J}_{A_{3}}
\end{array}\right] \\
\mathbf{J}_{B} & =\left[\begin{array}{lll}
\mathbf{J}_{B_{1}} & \mathbf{J}_{B_{2}} & \mathbf{J}_{B_{3}}
\end{array}\right] \\
\mathbf{J}_{C} & =\left[\begin{array}{lll}
\mathbf{J}_{C_{1}} & \mathbf{J}_{C_{2}} & \mathbf{J}_{C_{3}}
\end{array}\right] \\
\mathbf{J} & =\left[\begin{array}{lll}
\mathbf{j}_{1} & \mathbf{j}_{2} & \mathbf{j}_{3}
\end{array}\right] \\
\mathbf{J}_{l} & =\left[\begin{array}{lll}
\mathbf{j}_{l_{1}} & \mathbf{j}_{l_{2}} & \mathbf{j}_{l_{3}}
\end{array}\right]
\end{aligned}
$$

The $3 \times 2$ matrices $\mathbf{J}_{A_{i}}, \mathbf{J}_{B_{i}}$ and $\mathbf{J}_{C_{i}}$ and the three dimensional vectors $\mathbf{j}_{i}$ and $\mathbf{j}_{l_{i}}$ are expressed as :

$$
\begin{aligned}
\mathbf{J}_{A_{i}}=\left[\begin{array}{l}
\mathbf{j}_{A_{i} \phi} \\
\mathbf{J}_{A_{i} p}
\end{array}\right], \quad i=1,2,3 \\
\mathbf{J}_{B_{i}}=\left[\begin{array}{l}
\mathbf{j}_{B_{i} \phi} \\
\mathbf{J}_{B_{i} p}
\end{array}\right], \quad i=1,2,3 \\
\mathbf{J}_{C_{i}}=\left[\begin{array}{l}
\mathbf{j}_{C_{i} \phi} \\
\mathbf{J}_{C_{i} p}
\end{array}\right], \quad i=1,2,3 \\
\mathbf{j}_{i}=\left[\begin{array}{l}
j_{i \phi} \\
\mathbf{j}_{i p}
\end{array}\right], \quad i=1,2,3 \\
\mathbf{j}_{l_{i}}=\left[\begin{array}{l}
j_{l_{i} \phi} \\
\mathbf{j}_{l_{i} p}
\end{array}\right], \quad i=1,2,3
\end{aligned}
$$

$$
\begin{aligned}
\mathbf{j}_{A_{i} \phi} & =\frac{1}{\operatorname{det}(\mathbf{A})}\left[\begin{array}{ll}
v_{i} o_{i} & v_{i} p_{i}
\end{array}\right] \\
\mathbf{j}_{B_{i} \phi} & =\frac{1}{\operatorname{det}(\mathbf{A})}\left[\begin{array}{ll}
v_{i} q_{i} & v_{i} r_{i}
\end{array}\right]
\end{aligned}
$$

with

$$
\begin{aligned}
\mathbf{j}_{C_{i} \phi} & =\frac{1}{\operatorname{det}(\mathbf{A})}\left[\begin{array}{ll}
v_{i} s_{i} & v_{i} t_{i}
\end{array}\right] \\
j_{i \phi} & =\frac{v_{i} l_{i} \mathbf{v}_{i}^{T} \mathbf{u}_{i}}{\operatorname{det}(\mathbf{A})} \\
j_{l_{i} \phi} & =\frac{l_{i} v_{i}}{\operatorname{det}(\mathbf{A})}
\end{aligned}
$$

$$
\begin{aligned}
& \mathbf{J}_{A_{i} p}=\frac{1}{\operatorname{det}(\mathbf{A})}\left[\begin{array}{cc}
o_{i} \mathbf{w}_{i}^{T} \mathbf{i} & p_{i} \mathbf{w}_{i}^{T} \mathbf{i} \\
o_{i} \mathbf{w}_{i}^{T} \mathbf{j} & p_{i} \mathbf{w}_{i}^{T} \mathbf{j}
\end{array}\right] \\
& \mathbf{J}_{B_{i} p}=\frac{1}{\operatorname{det}(\mathbf{A})}\left[\begin{array}{cc}
q_{i} \mathbf{w}_{i}^{T} \mathbf{i} & r_{i} \mathbf{w}_{i}^{T} \mathbf{i} \\
q_{i} \mathbf{w}_{i}^{T} \mathbf{j} & r_{i} \mathbf{w}_{i}^{T} \mathbf{j}
\end{array}\right] \\
& \mathbf{J}_{C_{i} p}=\frac{1}{\operatorname{det}(\mathbf{A})}\left[\begin{array}{cc}
s_{i} \mathbf{w}_{i}^{T} \mathbf{i} & t_{i} \mathbf{w}_{i}^{T} \mathbf{i} \\
s_{i} \mathbf{w}_{i}^{T} \mathbf{j} & t_{i} \mathbf{w}_{i}^{T} \mathbf{j}
\end{array}\right] \\
& \mathbf{j}_{i p}=\frac{1}{\operatorname{det}(\mathbf{A})}\left[\begin{array}{c}
l_{i} \mathbf{v}_{i}^{T} \mathbf{u}_{i} \mathbf{w}_{i}^{T} \mathbf{i} \\
l_{i} \mathbf{v}_{i}^{T} \mathbf{u}_{i} \mathbf{w}_{i}^{T} \mathbf{j}
\end{array}\right] \\
& \mathbf{j}_{l_{i p}}=\frac{1}{\operatorname{det}(\mathbf{A})}\left[\begin{array}{c}
l_{i} \mathbf{w}_{i}^{T} \mathbf{i} \\
l_{i} \mathbf{w}_{i}^{T} \mathbf{j}
\end{array}\right]
\end{aligned}
$$

$o_{i}, p_{i}, q_{i}, r_{i}, s_{i}$ and $t_{i}$ taking the form:

$$
o_{i}=l_{i} \mathbf{v}_{i}^{T} \mathbf{i}
$$




$$
\begin{aligned}
p_{i} & =l_{i} \mathbf{v}_{i}^{T} \mathbf{j} \\
q_{i} & =-l_{i} \mathbf{v}_{i}^{T} \mathbf{E} \mathbf{u}_{i} \sin \theta_{i} \\
r_{i} & =l_{i} \mathbf{v}_{i}^{T} \mathbf{E} \mathbf{u}_{i} \cos \theta_{i} \\
s_{i} & =l_{i} \mathbf{v}_{i}^{T} \mathbf{k}_{i} \cos \beta_{i}-l_{i} \mathbf{v}_{i}^{T} \mathbf{E} \mathbf{k}_{i} \sin \beta_{i} \\
t_{i} & =l_{i} \mathbf{v}_{i}^{T} \mathbf{k}_{i} \sin \beta_{i}+l_{i} \mathbf{v}_{i}^{T} \mathbf{E} \mathbf{k}_{i} \cos \beta_{i}
\end{aligned}
$$

$\mathbf{j}_{A_{i} \phi}, \mathbf{j}_{B_{i} \phi}, \mathbf{j}_{C_{i} \phi}, j_{i \phi}$ and $j_{l_{i} \phi}$ contain the sensitivity coefficients of the MP orientation to variations in the Cartesian coordinates of points $A_{i}, B_{i}, C_{i}$, in the prismatic actuated variables $\rho_{i}$ and in length $l_{i}$, respectively. Similarly, $\mathbf{J}_{A_{i} p}, \mathbf{J}_{B_{i} p}, \mathbf{J}_{C_{i} p}, \mathbf{j}_{i p}$ and $\mathbf{j}_{l_{i} p}$ contain the sensitivity coefficients related to the MP position. It is apparent that this methodology can be applied to any PPM to obtain their sensitivity coefficients.

Finally, the designer of such PPMs can easily identify the most influential geometric variations to their MP pose and choose the proper dimensional tolerances from their sensitivity coefficients. Two aggregate sensitivity indices related to variations in the geometric errors of the moving and the base platforms are introduced thereafter.

\subsection{Two Aggregate Sensitivity Indices}

This section aims at determining indices in order to compare distinct PPMs with regard to the sensitivity of the pose of their moving platform to variations in their geometric parameters. What we mean by "distinct" PPMs is that they are different in terms of architecture and size. To this end, the relation between the MP pose and the variations in the geometric parameters is given by:

$$
\left[\begin{array}{c}
\delta \phi \\
\delta \mathbf{p}
\end{array}\right]=\mathbf{J}_{s_{M}} \mathbf{v}_{M}
$$

The $3 \times n_{M}$ matrix $\mathbf{J}_{s_{M}}$ is named "aggregate sensitivity Jacobian matrix" of manipulator $M$, and $n_{M}$ is the number of geometric variations that are considered. Assuming that actuated joints are not geometric parameters, $n_{3-R P R}$ is equal to 12 whereas $n_{3-R R R}$ and $n_{3-P R R}$ are equal to 18. The $n_{M}$-dimensional vector $\mathbf{v}_{M}$ contains the variations in the geometric parameters. The global sensitivity Jacobian matrices of the five PPMs under study can be expressed as follows:

$$
\begin{aligned}
\mathbf{J}_{s_{\underline{R} P R}} & =\left[\begin{array}{ll}
\mathbf{J}_{A_{\underline{R} P R}} & \mathbf{J}_{C_{\underline{R} P R}}
\end{array}\right] \\
\mathbf{J}_{s_{R \underline{P} R}} & =\left[\begin{array}{lll}
\mathbf{J}_{A_{R \underline{P} R}} & \mathbf{J}_{C_{R \underline{P} R}}
\end{array}\right] \\
\mathbf{J}_{s_{\underline{R} R R}} & =\left[\begin{array}{llll}
\mathbf{J}_{A_{\underline{R} R R}} & \mathbf{J}_{l_{1 \underline{R} R R}} & \mathbf{J}_{l_{2 \underline{R} R R}} & \mathbf{J}_{C_{\underline{R} R R}}
\end{array}\right] \\
\mathbf{J}_{s_{R \underline{R} R}} & =\left[\begin{array}{llll}
\mathbf{J}_{A_{R \underline{R} R}} & \mathbf{J}_{l_{1 R \underline{R} R}} & \mathbf{J}_{l_{2 R \underline{R} R}} & \mathbf{J}_{C_{R \underline{R} R}}
\end{array}\right] \\
\mathbf{J}_{s_{\underline{P} R R}} & =\left[\begin{array}{llll}
\mathbf{J}_{A_{\underline{P} R R}} & \mathbf{J}_{B_{\underline{P} R R}} & \mathbf{J}_{l_{\underline{P} R R}} & \mathbf{J}_{C_{\underline{P} R R}}
\end{array}\right]
\end{aligned}
$$


and

$$
\begin{aligned}
& \mathbf{v}_{\underline{R} P R}=\mathbf{v}_{R \underline{P} R}=\left[\begin{array}{ll}
\delta \mathbf{a}_{i} & \delta \mathbf{c}_{i}
\end{array}\right]^{T} \\
& \mathbf{v}_{\underline{R} R R}=\mathbf{v}_{R \underline{R} R}=\left[\begin{array}{llll}
\delta \mathbf{a}_{i} & \delta \mathbf{l}_{1 i} & \delta \mathbf{l}_{2 i} & \delta \mathbf{c}_{i}
\end{array}\right]^{T} \\
& \mathbf{v}_{\underline{P} R R}=\left[\begin{array}{llll}
\delta \mathbf{a}_{i} & \delta \mathbf{b}_{i} & \delta \mathbf{l}_{i} & \delta \mathbf{c}_{i}
\end{array}\right]^{T}
\end{aligned}
$$

with

$$
\begin{aligned}
& \delta \mathbf{a}_{i}=\left[\begin{array}{llllll}
\delta a_{1 x} & \delta a_{1 y} & \delta a_{2 x} & \delta a_{2 y} & \delta a_{3 x} & \delta a_{3 y}
\end{array}\right] \\
& \delta \mathbf{c}_{i}=\left[\begin{array}{llllll}
\delta c_{1 X} & \delta c_{1 Y} & \delta c_{2 X} & \delta c_{2 Y} & \delta c_{3 X} & \delta c_{3 Y}
\end{array}\right] \\
& \delta \mathbf{l}_{1 i}=\left[\begin{array}{lll}
\delta l_{11} & \delta l_{12} & \delta l_{13}
\end{array}\right] \\
& \delta \mathbf{l}_{2 i}=\left[\begin{array}{lll}
\delta l_{21} & \delta l_{22} & \delta l_{23}
\end{array}\right] \\
& \delta \mathbf{b}_{i}=\left[\begin{array}{llllll}
\delta b_{1 x} & \delta b_{1 y} & \delta b_{2 x} & \delta b_{2 y} & \delta b_{3 x} & \delta b_{3 y}
\end{array}\right] \\
& \delta \mathbf{l}_{i}=\left[\begin{array}{lll}
\delta l_{1} & \delta l_{2} & \delta l_{3}
\end{array}\right]
\end{aligned}
$$

The $3 \times n_{M}$ matrices $\mathbf{J}_{s_{M}}$ is composed of two blocks, $\mathbf{j}_{s_{M} \phi}$ and $\mathbf{J}_{s_{M}}$, i.e.,

$$
\mathbf{J}_{s_{M}}=\left[\begin{array}{l}
\mathbf{j}_{s_{M} \phi} \\
\mathbf{J}_{s_{M} p}
\end{array}\right]
$$

The expressions of $\mathbf{j}_{s_{M} \phi}$ and $\mathbf{j}_{s_{M} p}$ are given in Appendix 1. The sensitivity matrices of the 3RPR PPM and the 3-RRR PPM are given in [7, 9]. From Eq.(20) and Appendix 1, we define an aggregate sensitivity index $\nu_{\phi_{M}}$ of the MP orientation to variations in the geometric parameters:

$$
\nu_{\phi_{M}}=\frac{\left\|\mathbf{j}_{s_{M} \phi}\right\|_{2}}{n_{M}}
$$

Likewise, an aggregate sensitivity index $\nu_{p_{M}}$ of the MP position to variations in its geometric parameters is defined as:

$$
\nu_{p_{M}}=\frac{\left\|\mathbf{J}_{s_{M} p}\right\|_{2}}{n_{M}}
$$

$\|$. $\|_{2}$ denotes the Euclidean norm. The lower $\nu_{\phi_{M}}$, the lower the aggregate sensitivity of the orientation of the MP of the manipulators to variations in its geometric parameters. Similarly, the lower $\nu_{p_{M}}$, the lower the aggregate sensitivity of the position of the MP to variations in the geometric parameters. As a matter of fact, $\nu_{\phi_{M}}\left(\nu_{p_{M}}\right.$, resp.) characterizes the intrinsic sensitivity of the orientation (position, resp.) of the MP to any variation in the geometric parameters.

Let us notice that $\nu_{p_{M}}$ as well as the sensitivity coefficients related to the MP position defined in this Section and Section 3.1 are frame dependent, whereas $\nu_{\phi_{M}}$ and the sensitivity coefficients related to the MP orientation are not.

Finally, let us notice that $\nu_{\phi_{M}}$ indices are expressed in $[\mathrm{rad} / \mathrm{L}]$, whereas $\nu_{p_{M}}$ indices are 
dimensionless, [L] being the unit of length.

\section{Comparison Methodology}

In this section, we define a methodology to compare PPMs with regard to their workspace size and sensitivity. This methodology is broken down into six steps:

1. normalization of the geometric parameters;

2. determination of the manipulator workspace (WS) and the regular workspace (RW);

3. determination of the smallest regular workspace $\left(R W_{\min }\right)$;

4. evaluation of the sensitivity of the MP orientation to variations in the geometric parameters throughout the $R W_{\min }$ by means of $\nu_{\phi_{M}}$ defined in Eq.(??);

5. evaluation of the sensitivity of the MP position to variations in the geometric parameters throughout the $R W_{\min }$ by means of $\nu_{p_{M}}$ defined in Eq.(??);

6. comparison with the average and the maximum sensitivities of the manipulator throughout its $R W_{\text {min }}$.

The radii of the circumcircles of the base and the moving platforms of the manipulators are normalized as explained in Section ??. The dimensions of the legs and the passive and actuated joints are determined in such a way that the manipulators under study have equivalent workspaces. The RW of the manipulators are obtained by means of an optimization problem introduced in Section ??. Finally, the smallest one is taken $\left(R W_{\min }\right)$, and the sensitivity is evaluated thoughout $R W_{\text {min }}$.

\subsection{Geometric Parameters Normalization}

Let $R_{1}$ and $R_{2}$ be the radii of the base and moving platforms of the PPM. In order to come up with finite values, $R_{1}$ and $R_{2}$ are normalized as in [14, 15, 16]. For that matter, let $N_{f}$ be a normalizing factor:

$$
N_{f}=\left(R_{1}+R_{2}\right) / 2
$$

and

$$
r_{m}=R_{m} / N_{f}, \quad m=1,2
$$

Therefore,

$$
r_{1}+r_{2}=2
$$

From eq.(??), we can notice that:

$$
r_{1} \in[0,2], r_{2} \in[0,2]
$$


Moreover, the circumcircle radii of the base and moving platforms, i.e., $r_{1}$ and $r_{2}$, are similar for the manipulators under study. All PPMs are symmetrical, i.e., the base and moving platforms are equilateral. Consequently, we defined ratio $r_{1} / r_{2}$ and the other geometric parameters as follows :

$$
\begin{aligned}
r_{2} / r_{1} & =\frac{1}{5} \\
a_{1} & =a_{2}=a_{3}=r_{1}=\frac{5}{3} \\
c_{1} & =c_{2}=c_{3}=r_{2}=\frac{1}{3} \\
\left\{\alpha_{1}, \alpha_{2}, \alpha_{3}\right\} & =\{-5 \pi / 6,-\pi / 6, \pi / 2\} \\
\left\{\beta_{1}, \beta_{2}, \beta_{3}\right\} & =\{-5 \pi / 6,-\pi / 6, \pi / 2\}
\end{aligned}
$$

As the former two-dimensional infinite space corresponding to geometric parameters $R_{1}$ and $R_{2}$ is reduced to a one-dimensional finite space defined with Eqs.(??) and (??), the workspace analysis of the PPM under study is easier. Moreover, $\alpha_{i}$ and $\beta_{i}, i=1,2,3$, are given in [rad] and $r_{1}$ and $r_{2}$ are given in [m]. It is apparent that the base and the moving platforms are equilateral. For the 3-RRR PPMs,

$$
\begin{aligned}
l_{11} & =l_{12}=l_{13}=l_{21}=l_{22}=l_{23}=l \\
l & =\frac{-r_{2}+\sqrt{r_{2}^{2}+2\left(r_{1}^{2}-r_{2}^{2}\right)}}{2}
\end{aligned}
$$

$l$ is obtained in such a way that $l_{1 i}$ and $l_{2 i}, i=1,2,3$, are identical and to have the same workspace size for the 3 - $\underline{R R R}$ and the 3 -R $\underline{R R}$ PPMs [17]. Moreover, we can determine an isotropic configuration for each 3-RRR. In an isotropic configuration, the sensitivity of a manipulator in both velocity and force or torque errors is a minimum, and the manipulator can be controlled equally well in all directions. The concept of kinematic isotropy has been used as a criterion in the design of planar manipulators [18]. The actuated joints limits are:

$$
\begin{aligned}
& 0<\rho_{i R P R}<2 l \\
& 0<\rho_{i P R R}<2 l
\end{aligned}
$$

For the 3-PRR PPM,

$$
l_{1}=l_{2}=l_{3}=l
$$

With the geometric parameters normalization the PPMs under study have an equivalent size. Finally, each PPM has an isotropic configuration. The sensitivity analysis is conducted in the vicinity of the isotropic configuration. We define an isotropic pose for every PPM, the position is the same, i.e., $\mathbf{p}=[0,0]$ but the orientation is different. The orientation $\phi_{i s o M}$ corresponding to an isotropic configuration of manipulator $M$ is given below:

$$
\phi_{i s o \underline{R} P R}=0
$$




$$
\begin{aligned}
& \phi_{i s o R \underline{P} R}=\cos ^{-1}\left(\frac{r_{2}}{r_{1}}\right) \\
& \phi_{i s o \underline{R} R R}=\frac{\pi}{4}-\cos ^{-1}\left(\frac{r_{1}^{2}+2 l^{2}-r_{2}^{2}}{2 \sqrt{2} l r_{1}}\right) \\
& \phi_{i s o R \underline{R} R}=\cos ^{-1}\left(\frac{r_{1}^{2}+2 l^{2}-r_{2}^{2}}{2 \sqrt{2} l r_{1}}\right)-\frac{\pi}{4} \\
& \phi_{i s o \underline{P} R R}=\frac{5 \pi}{6}-\cos ^{-1}\left(\frac{l}{\sqrt{r_{2}^{2}+l^{2}}}\right)-\cos ^{-1}\left(\frac{r_{1}}{2 \sqrt{r_{2}^{2}+l^{2}}}\right)
\end{aligned}
$$

\subsection{Regular Workspace}

Assessing the kinetostatic performance of parallel manipulators is not an easy task for 6-DOF parallel manipulators [19]. However, it is easier for planar manipulators as their singularities have a simple geometric interpretation [20, 21]. The RW of a manipulator is a regular-shaped part of its workspace with good and homogeneous kinetostatic performance. The shape of the RW is up to the designer. It may be a cube, a parallelepiped, a cylinder or another regular shape. A reasonable choice is a shape that fits well the one of the singular surfaces. It appears that a cylinder suits well for planar manipulators.

In the scope of this study, let the RW of the PPM be a cylinder of $\phi$-axis with a good kinetostatic performance, i.e., the orientation range $\Delta \phi$ is defined around $\phi_{i s o M}$ and the sign of the determinant of the kinematic Jacobian matrix of the manipulator, i.e., $\operatorname{sign}(\operatorname{det}(\mathbf{J})$ ), remains constant. Accordingly, the RW of the PPMs are obtained by solving the following optimization problem:

$$
P b \mid \begin{array}{ll}
\text { minimize } & 1 / R \\
\text { over } & \mathbf{x}=\left[\begin{array}{lllll}
R & I_{x} & I_{y} & \phi_{\min } & \phi_{\max }
\end{array}\right] \\
\text { subject to } & \Delta \phi \geq \pi / 6 \\
& \operatorname{sign}(\operatorname{det}(\mathbf{J}))=\text { constant }
\end{array}
$$

$R$ is the radius of the cylinder and $\Delta \phi$ the orientation range of the MP of the manipulator within its RW. Here, $\Delta \phi$ is set to $\pi / 6$ arbitrarily. This optimization problem has five decision variables, namely, $\mathbf{x}=\left[\begin{array}{lllll}R & I_{x} & I_{y} & \phi_{\min } & \phi_{\max }\end{array}\right] . I_{x}$ and $I_{y}$ are the Cartesian coordinates of the center of the cylinder. $\phi_{\min }$ and $\phi_{\max }$ are the lower and upper bounds of $\Delta \phi$ and are defined as follows:

$$
\begin{aligned}
\phi_{\text {min }} & =\phi_{\text {isoM }}-\frac{\Delta \phi}{2} \\
\phi_{\max } & =\phi_{i s o M}+\frac{\Delta \phi}{2}
\end{aligned}
$$

The global minimum, i.e., the optimum RW of the manipulator, of problem (21) is obtained by means of a Tabu search Hooke and Jeeves algorithm [22]. Finally, $\nu_{\phi_{M}}$ and $\nu_{p_{M}}$ are used to evaluate the global orientation and positioning errors of the manipulator throughout the RW of the PPMs under study. 


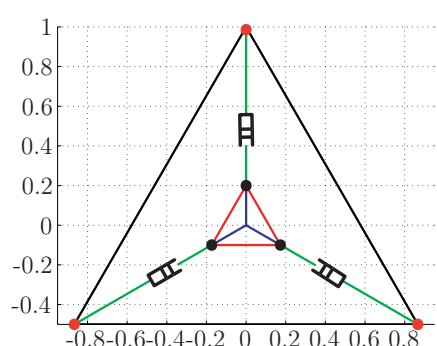

(a) 3 - $\underline{R P R}$

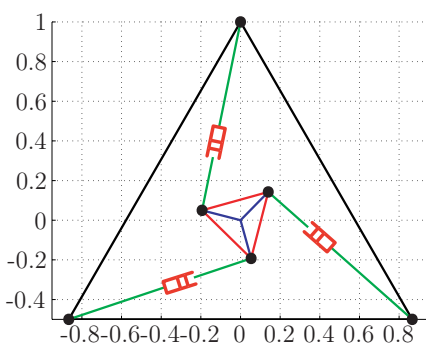

(b) 3 -R $\underline{P R}$

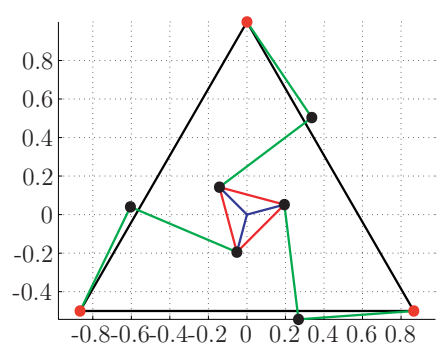

(c) 3 - $\underline{R R R}$

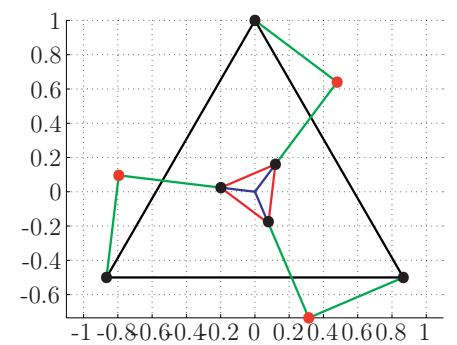

(d) 3-RR

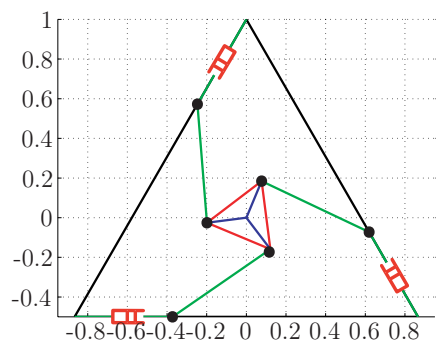

(e) 3 - $\underline{P R R}$

Figure 2: Manipulators under study is an isotropic configurations

\section{Illustrative Examples: Comparison of Five PPMs}

This section aims at illustrating the sensitivity indices and comparison methodology introduced in Sections 3.2 and 4 , respectively. For that purpose, the sensitivity of the symmetrical (base and MP are equilateral) 3- $\underline{R P R}, 3-R \underline{P R}, 3-\underline{R R R}, 3-R \underline{R R}$ and 3-PRR PPMs are analyzed in detail. Then, their sensitivity are compared.

\subsection{Sensitivity Analysis}

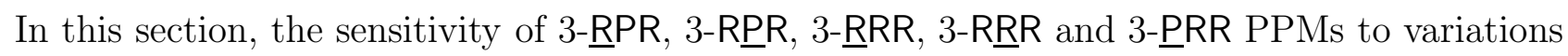
in their geometric parameters is evaluated within their WS for a matter of comparison based on the aggregate sensitivity indices $\nu_{\phi_{M}}$ and $\nu_{p_{M}}$ defined in Eqs.(??) and (??), respectively. Figures 2(a)-(e) illustrate the corresponding manipulators, before geometric parameters normalization, the radii of the circumscribed circles of their base and moving platforms being different. The PPMs are represented in their isotropic configuration, the orientation $\phi$ of their MP being equal to $\phi_{i s o M}$ and point $P$ being coincident with the origin of $\mathcal{F}_{b}$, i.e., $\mathbf{p}=[0,0]^{T}$.

In order to have an idea of the aggregate sensitivity of the MP pose of the manipulator to variations in its geometric parameters, Figs. 3(a) to 7)(b) illustrate the isocontours of $\nu_{\phi_{M}}$ and $\nu_{p_{M}}$, for a given orientation range $\Delta \phi$ centered at $\phi_{i s o M}$ of the MP throughout the WS of the 3- $\underline{R P R}, 3-\mathrm{R} \underline{P R}, 3-\underline{R R R}, 3-\mathrm{R} \underline{R R}$ and 3-PRR PPMs, respectively. We can notice that the closer $P$ to the geometric center of WS, the larger the aggregate sensitivity of the MP pose to variations in the geometric parameters. It is apparent that the orientation and the position of the MP of the 3 - $\underline{R} P R$ is the most sensitive to variations in geometric parameters.

It appears that the two aggregate sensitivity indices can be used as amplification factors of any geometric parameter error of the PPMs. 


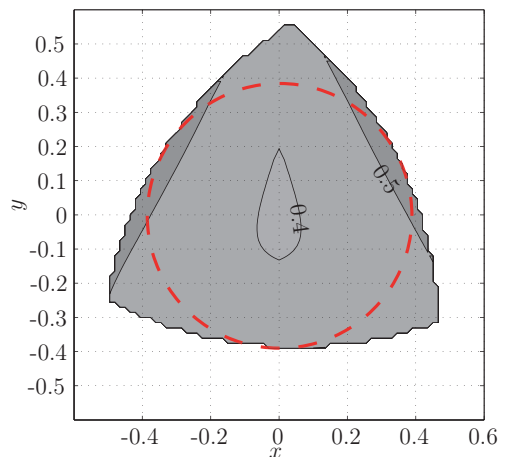

(a) $\nu_{\phi_{\underline{R} P R}}$

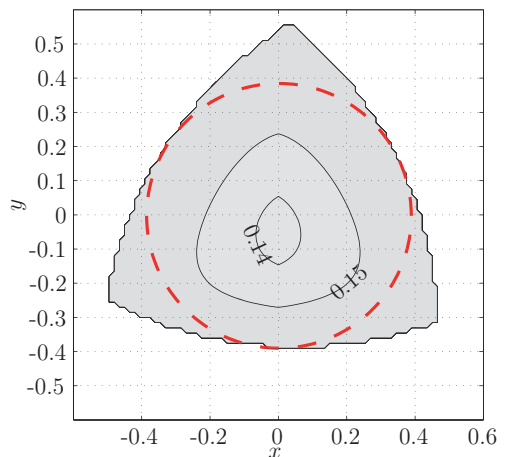

(b) $\nu_{p_{\underline{R} P R}}$

Figure 3: Sensitivity isocontours of the 3-RPR manipulator

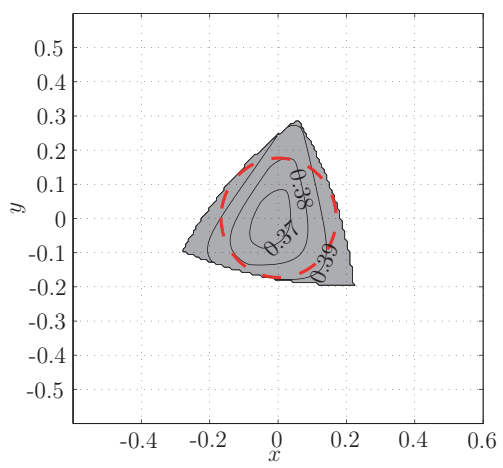

(a) $\nu_{\phi_{R \underline{P} R}}$

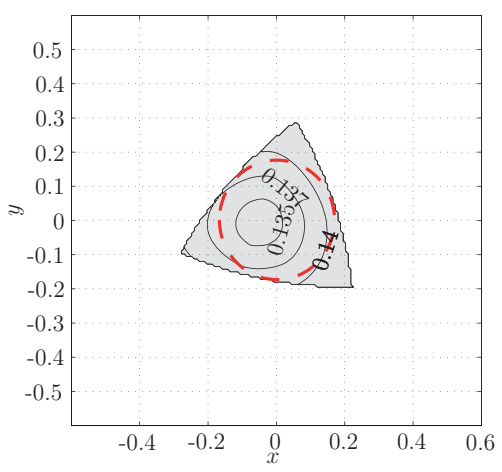

(b) $\nu_{p_{R \underline{P} R}}$

Figure 4: Sensitivity isocontours of the 3-RR manipulator

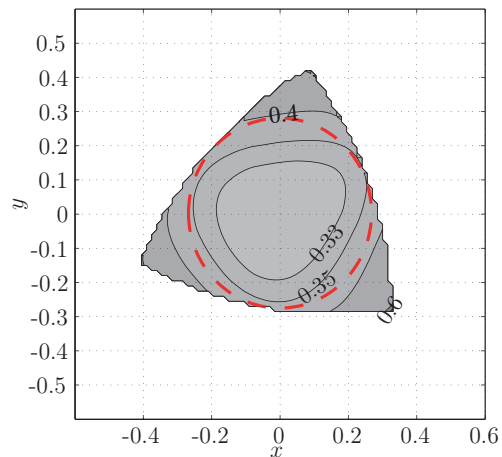

(a) $\nu_{\phi_{\underline{R} R R}}$

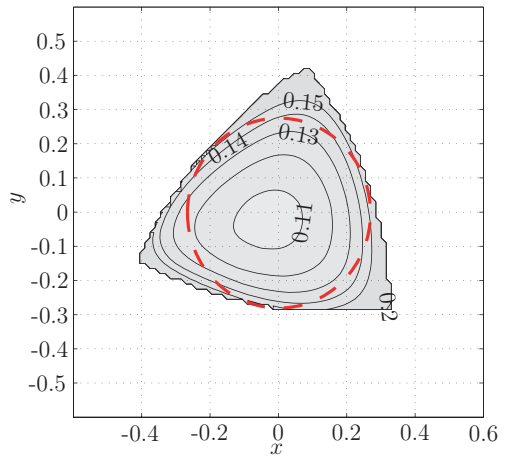

(b) $\nu_{p_{\underline{R} R R}}$

Figure 5: Sensitivity isocontours of the 3-RRR manipulator 


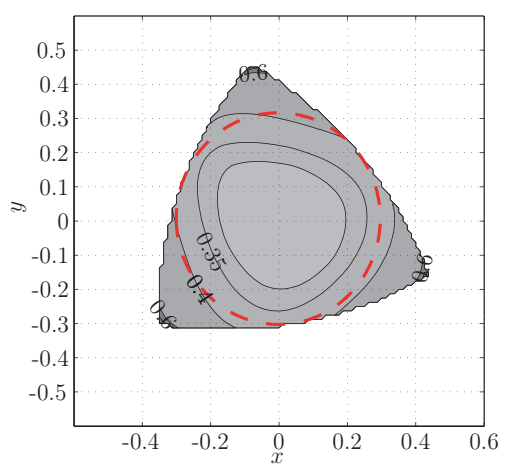

(a) $\nu_{\phi_{R} \underline{R} R}$

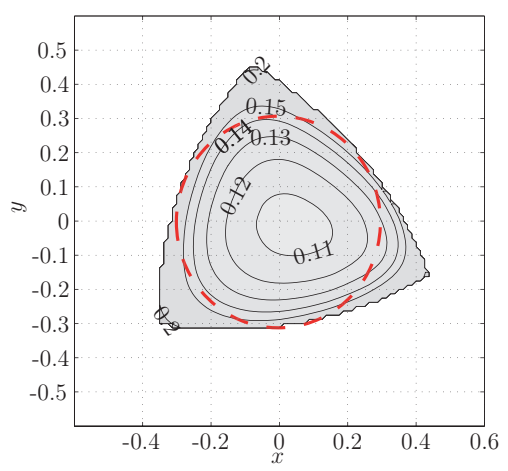

(b) $\nu_{p_{R} \underline{R}}$

Figure 6: Sensitivity isocontours of the 3-RR manipulator

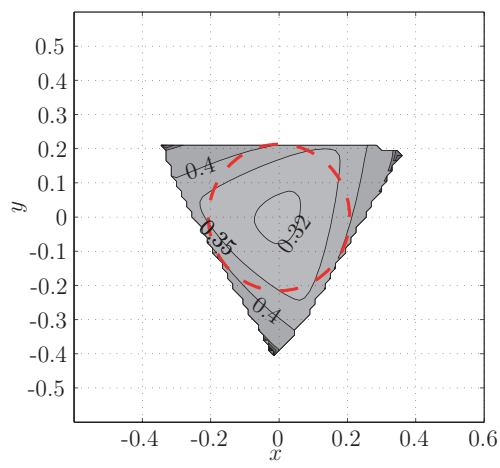

(a) $\nu_{\phi_{\underline{P} R R}}$

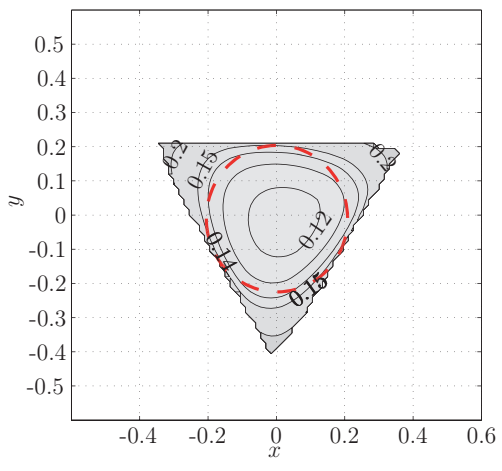

(b) $\nu_{p_{\underline{P} R R}}$

Figure 7: Sensitivity isocontours of the 3-PRR manipulator

\subsection{Comparative study}

In order to highlight the comparison methodology proposed in Section 4, we used the sensitivity analysis illustrated in Section ??. Whether they are globally more or less sensitive to geometric errors than their PPMs counterparts is a question of interest for the designer. In order to compare the sensitivity of the foregoing manipulators, we first define their Regular Workspace (RW). Then, the sensitivity of the 3 - $\underline{R P R}, 3-\mathrm{R} \underline{P R}, 3-\underline{R R R}, 3-\mathrm{R} \underline{R} \mathrm{R}$ and $3-\underline{P R R} \mathrm{PPMs}$ can be evaluated and compared throughout the smallest RW. Their radii are illustrated in Figs. 3(a) to $7(\mathrm{~b})$ in red circle dashed and are given in Table ?? and compared. We can notice that the

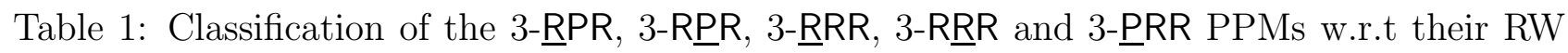
size

\begin{tabular}{c|ccccc}
\hline \hline & $3-\underline{R P R}$ & $3-\mathrm{R} \underline{\mathrm{PR}}$ & $3-\underline{\mathrm{R} R R}$ & $3-\mathrm{R} \underline{\mathrm{R} R}$ & $3-\underline{\mathrm{PRR}}$ \\
\hline$R W$ & 0.387 & 0.177 & 0.272 & 0.272 & 0.206 \\
\hline \hline
\end{tabular}

3-RPR PPMs have the largest RW, whereas the 3-RPR have the smallest RW. Therefore, we use the 3-RPR RW, called $R W_{\min }$ to evaluate the average and the maximum sensitivities of each PPM under study. 


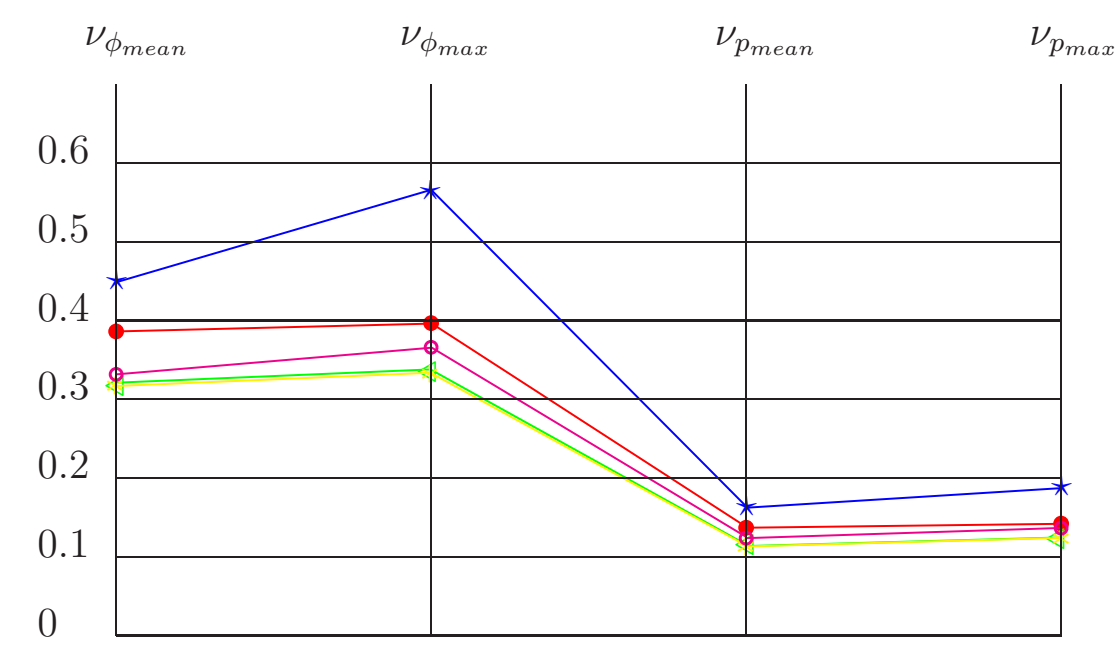

Figure 8: Sensitivity indices values : $\star \star \star: 3-\underline{R P R}, \bullet \cdots: 3-\mathrm{R} \underline{\mathrm{PR}}, \triangleleft \triangleleft: 3-\underline{\mathrm{R} R R}, *-*: 3-\mathrm{R} \underline{\mathrm{R}}$, o- o: $3-\underline{P R R}$

Finally, Table ?? gives the sensitivity results of $3-\underline{R P R}, 3-\mathrm{R} \underline{\mathrm{PR}}, 3-\underline{R} R R$, 3-RR $\underline{R}$ and $3-\underline{P} R R$ PPMs with regard to their average and maximum sensitivity of the orientation and the position of their MP to variations in their geometric parameters, throughout $R W_{\min }$. The results are quite similar and good, because the sensitivity analysis is evaluated in their workspace center $\left(R W_{\min }\right)$ and around their isotropic orientation $\left(\phi_{i s o M}\right)$. In addition, the two aggregate sensitivity indices can be considered as mean amplification factors of any geometric parameter error of the PPMs. Hence, with these results, there is no error amplification. However, these results are illustrated in Fig. 8 and we can notice that the 3-RPR manipulator is globally the least interesting, i.e., it has the least robust design. Finally, the position of point $P$ on the moving platform affects the shape of the sensitivity isocontours and the global sensitivity indices $\nu_{\phi}$ and $\nu_{p}$, but does not change the results of the previous comparison.

Table 2: Mean and maximum global sensitivity indices $\nu_{\phi}$ and $\nu_{p}$

\begin{tabular}{c|ccccc}
\hline \hline & $3-\underline{\mathrm{RPR}}$ & $3-\mathrm{RPR}$ & $3-\underline{\mathrm{RRR}}$ & $3-\mathrm{RRR}$ & $3-\underline{\mathrm{PRR}}$ \\
\hline$\nu_{\phi_{\text {mean }}}$ & 0.4487 & 0.3866 & 0.3211 & 0.3172 & 0.3321 \\
\hline$\nu_{\phi_{\text {max }}}$ & 0.5664 & 0.3969 & 0.3377 & 0.3337 & 0.3662 \\
\hline$\nu_{p_{\text {mea }}}$ & 0.1626 & 0.1372 & 0.1138 & 0.1134 & 0.1238 \\
\hline$\nu_{p_{\text {max }}}$ & 0.1881 & 0.1423 & 0.1244 & 0.1242 & 0.1368 \\
\hline \hline
\end{tabular}

\section{Conclusions}

This paper dealt with the sensitivity comparison of five planar parallel manipulators, namely, the 3-RPR, 3-R $\underline{P R}, 3-\underline{R R R}, 3-\mathrm{R} \underline{R}$ and 3-PRR planar parallel manipulators. First, we have introduced a methodology to obtain the sensitivity coefficients of the orientation and the position of the moving platform of the planar parallel manipulators to variations in their geometric 
parameters and actuated variables. Their sensitivity coefficients were derived and expressed algebraically. Moreover, two aggregate sensitivity indices were determined for each manipulator under study, one related to the orientation of the moving platforms of the manipulator and another one related to their position. Then, a methodology was proposed to compare planar parallel manipulators with regard to their workspace size and sensitivity. Finally, the sensitivity

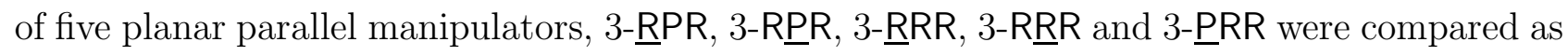
illustrative examples. The sensitivity indices $\nu_{\phi_{M}}$ and $\nu_{p_{M}}$ introduced in the paper should help the designer of planar parallel manipulators at the conceptual design stage. Joint clearances and flexibilities also affect the positioning accuracy. The sensitivity to joint clearances and flexibilities in the revolute joints can be taken into account in the definition of the variations in the positions of the revolute joint centers. Prismatic joint clearances and link flexibilities will be studied in future work, considering also spatial manipulators.

\section{References}

[1] Yu, A., Bonev, I. and Zsombor-Murray P. (2009). "Geometric approach to the accuracy analysis of a class of 3-DOF planar parallel robots," Mechanism and Machine Theory, 43(3), pp. 364-375.

[2] Kim, H.S., and Tsai, L-W., (2003). "Design optimization of a Cartesian parallel manipulator," ASME Journal of Mechanical Design, 125, pp. 43-51.

[3] Bai, S. and Caro, S. (2009). "Design and Analysis of a 3-PPR Planar Robot with U-shape Base", Proceedings of the 14th International Conference on Advanced Robotics, Munich, Germany.

[4] Briot, S. and Bonev, I.A. (2007). "Accuracy Analysis of 3-DOF Planar Parallel Robots," Mechanism and Machine Theory, 43, pp. 445-458.

[5] Merlet, J.P. (2006). Parallel robots, 2nd ed., Springer.

[6] Caro, S., Wenger, P., Bennis, F., and Chablat, D., (2006). "Sensitivity Analysis of the Orthoglide, A 3-DOF Translational Parallel Kinematic Machine," ASME Journal of Mechanical Design, 128, March, pp. 392-402.

[7] Caro, S., Binaud, N. and Wenger, P. (2009). "Sensitivity Analysis of 3-RPR Planar Parallel Manipulators," ASME Journal of Mechanical Design, 131, March, pp. 121005-1121005-13.

[8] Angeles, J., 2007. Fundamentals of Robotic Mechanical Systems, Theory, Methods, Algorithms, Third Edition, Springer, New York. (first edition published in 1997.)

[9] Binaud, N., Caro, S. and Wenger, P. (2009). "Sensitivity and Dexterity Comparison of 3-RRR planar parallel manipulators," 5th International Worshop on Computional Kinematics, Duisburg, Germany. 
[10] Cha, S.H., Lasky, T.A. and Velinsky, S.A. (2007). "Singularity Avoidance for the 3-RRR Mechanism Using Kinematic Redundancy," IEEE International Conference on Robotics and Automation, Roma, Italy.

[11] Firmani, F. and Podhorodeski R.P. (2009). "Singularity analysis of planar parallel manipulators based on forward kinematic solutions," Mechanism and Machine Theory, 44, pp. 1386-1399.

[12] Bonev, I.A., Zlatanov, D. and Gosselin, C.M. (2003). "Singularity Analysis of 3-DOF Planar Parallel Mechanisms via Screw Theory," Journal of Mechanical Design, 125, pp.573581.

[13] Masouleh, M.T. and Gosselin, C. (2007). "Determination of Singularity-Free Zones in the Workspace of Planar 3-PRR Parallel Mechanisms," Journal of Mechanical Design, 129, pp.649-652.

[14] Liu, X.-J., Wang, J. and Pritschow, G. (2006). "Kinematics, Singularity and Workspace of Planar 5R Symmetrical Parallel Mechanisms," Mechanism and Machine Theory, 41(2), pp. 145-169.

[15] Liu, X.-J., Wang, J. and Pritschow, G. (2006). "Performance Atlases and Optimum Design of Planar 5R Symmetrical Parallel Mechanisms," Mechanism and Machine Theory, 41(2), pp. 119-144.

[16] Liu, X.-J., Wang, J. and Pritschow, G. (2006). "On the Optimal Design of the PRRRP 2-DOF Parallel Mechanism," Mechanism and Machine Theory, 41(9), pp. 1111-1130.

[17] Alba-Gomez, O., Wenger, P. and Pamanes, A. (2005). "Consistent kinetostatic indices for planar 3-dof parallel manipulators, application to the optimal kinematic inversion," ASME International Design Engineering Technical Conferences and Computers and Information In Engineering Conference, Long Beach, California, USA.

[18] Gosselin, C. and Angeles, J. (1988). "The optimum kinematic design of a planar threedegree-of-freedom parallel manipulator," Trans. ASME J. Mech. Transm. Automat. Des., 110, pp. 3541.

[19] Merlet, J.P. (2006). "Jacobian, Manipulability, Condition Number, and Accuracy of Parallel Robots," ASME Journal of Mechanical Design, 128, pp. 199-206.

[20] Caro, S., Chablat, D., Wenger, P. and Angeles, J. (2003). "Isoconditioning Loci of Planar Three-Dof Parallel Manipulators," In: G. Gogu, D. Coutellier, P. Chedmail and P. Ray (Editors), Recent Advances in Integrated Design and Manufacturing in Mechanical Engineering, Kluwer Academic Publisher, pp. 129-138. 
[21] Alba-Gomez, O., Wenger, P. and Pamanes, A. (2005). "Consistent Kinetostatic Indices for Planar 3-DOF Parallel Manipulators, Application to the Optimal Kinematic Inversion," ASME Design Engineering Technical Conferences, September, Long Beach, U.S.A.

[22] Al-Sultan, K.S. and Al-Fawzan, M.A. (1997). "A Tabu Search Hooke and Jeeves Algorithm for Unconstrained Optimization," European Journal of Operational Research, 103, pp. 198-208.

\section{Appendices}

APPENDIX 1 : Sensitivity matrices for the manipulators under study

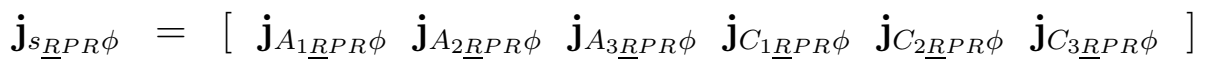

$$
\begin{aligned}
& \mathbf{j}_{s_{R \underline{P} R} \phi}=\left[\begin{array}{llllll}
\mathbf{j}_{A_{1 R \underline{P} R} \phi} & \mathbf{j}_{A_{2 R \underline{P} R} \phi} & \mathbf{j}_{A_{3 R \underline{P} R} \phi} & \mathbf{j}_{C_{1 R \underline{P} R} \phi} & \mathbf{j}_{C_{2 R \underline{P} R} \phi} & \mathbf{j}_{C_{3 R \underline{P} R} \phi}
\end{array}\right] \\
& \mathbf{j}_{s_{\underline{R} R R} \phi}=\left[\begin{array}{llllll}
\mathbf{j}_{A_{1 \underline{R} R R} \phi} & \mathbf{j}_{A_{2 \underline{R} R R} \phi} & \mathbf{j}_{A_{3 \underline{R} R R} \phi} & j_{l_{11 \underline{R} R R} \phi} & j_{l_{12 \underline{R} R R} \phi} & j_{l_{13 \underline{R} R R} \phi}
\end{array}\right. \\
& \left.\begin{array}{llllll}
j_{l_{2 \underline{R} R R} \phi} & j_{l_{22 \underline{R} R R} \phi} & j_{l_{2 \underline{R} R R} \phi} & \mathbf{j}_{C_{1 \underline{R} R R} \phi} & \mathbf{j}_{C_{2 \underline{R} R R} \phi} & \mathbf{j}_{C_{3 \underline{R} R R} \phi}
\end{array}\right] \\
& \mathbf{j}_{s_{R \underline{R} R} \phi}=\left[\begin{array}{llllll}
\mathbf{j}_{A_{1 R \underline{R} R} \phi} & \mathbf{j}_{A_{2 R \underline{R} R} \phi} & \mathbf{j}_{A_{3 R \underline{R} R} \phi} & j_{l_{11 R \underline{R} R} \phi} & j_{l_{12 R \underline{R} R} \phi} & j_{l_{13 R \underline{R} R} \phi}
\end{array}\right. \\
& \left.\begin{array}{llllll}
j_{l_{21 R \underline{R} R} \phi} & j_{l_{22 R \underline{R} R} \phi} & j_{l_{23 R \underline{R} R} \phi} & \mathbf{j}_{C_{1 R \underline{R} R} \phi} & \mathbf{j}_{C_{2 R \underline{R} R} \phi} & \mathbf{j}_{C_{3 R \underline{R} R} \phi}
\end{array}\right] \\
& \mathbf{j}_{\underline{\underline{P}}^{\prime} R R} \phi=\left[\begin{array}{llllll}
\mathbf{j}_{A_{1 \underline{P} R R} \phi} & \mathbf{j}_{A_{2 \underline{P} R R} \phi} & \mathbf{j}_{A_{3 \underline{P} R R} \phi} & \mathbf{j}_{B_{1 \underline{P} R R} \phi} & \mathbf{j}_{B_{2 \underline{P} R R} \phi} & \mathbf{j}_{B_{3 \underline{P} R R} \phi}
\end{array}\right. \\
& \left.\begin{array}{llllll}
j_{l_{1 \underline{P} R R} \phi} & j_{l_{2 \underline{P} R R} \phi} & j_{l_{\underline{B} R R} \phi} & \mathbf{j}_{C_{1 \underline{P} R R} \phi} & \mathbf{j}_{C_{2 \underline{P} R R} \phi} & \mathbf{j}_{C_{3 \underline{P} R R} \phi}
\end{array}\right] \\
& \mathbf{J}_{s_{\underline{R} P R} p}=\left[\begin{array}{llllll}
\mathbf{J}_{A_{1 \underline{R} P R} p} & \mathbf{J}_{A_{2 \underline{R} P R} p} & \mathbf{J}_{A_{3 \underline{R} P R} p} & \mathbf{J}_{C_{1 \underline{R} P R} p} & \mathbf{J}_{C_{2 \underline{R} P R} p} & \mathbf{J}_{C_{3 \underline{R} P R} p}
\end{array}\right] \\
& \mathbf{J}_{s_{R \underline{P} R} p}=\left[\begin{array}{llllll}
\mathbf{J}_{A_{1 R \underline{P} R} p} & \mathbf{J}_{A_{2 R \underline{P} R} p} & \mathbf{J}_{A_{3 R \underline{R} R} p} & \mathbf{J}_{C_{1 R \underline{P} R} p} & \mathbf{J}_{C_{2 R \underline{P} R} p} & \mathbf{J}_{C_{3 R \underline{P} R} p}
\end{array}\right] \\
& \mathbf{J}_{s_{\underline{R} R R} p}=\left[\begin{array}{llllll}
\mathbf{J}_{A_{1 \underline{R} R R} p} & \mathbf{J}_{A_{2 \underline{R} R R} p} & \mathbf{J}_{A_{3 \underline{R} R R} p} & \mathbf{j}_{l_{11 \underline{R} R R} p} & \mathbf{j}_{l_{12 \underline{R} R R} p} & \mathbf{j}_{l_{13 \underline{R} R R} p}
\end{array}\right. \\
& \left.\begin{array}{lllllll}
\mathbf{j}_{l_{21 \underline{R} R R} p} & \mathbf{j}_{l_{22 \underline{R} R R} p} & \mathbf{j}_{l_{2 \underline{R} R R} p} & \mathbf{J}_{C_{1 \underline{R} R R} p} & \mathbf{J}_{C_{2 \underline{R} R R} p} & \mathbf{J}_{C_{3 \underline{R} R R} p}
\end{array}\right] \\
& \mathbf{J}_{s_{R \underline{R} R} p}=\left[\begin{array}{llllll}
\mathbf{J}_{A_{1 R \underline{R} R} p} & \mathbf{J}_{A_{2 R \underline{R} R} p} & \mathbf{J}_{A_{3 R \underline{R} R} p} & \mathbf{j}_{l_{11 R \underline{R} R} p} & \mathbf{j}_{l_{12 R \underline{R} R} p} & \mathbf{j}_{l_{13 R \underline{R} R} p}
\end{array}\right. \\
& \left.\begin{array}{llllll}
\mathbf{j}_{l_{21 R \underline{R} R} p} & \mathbf{j}_{l_{22 R \underline{R} R} p} & \mathbf{j}_{23 R \underline{R} R} & \mathbf{J}_{C_{1 R \underline{R} R} p} & \mathbf{J}_{C_{2 R \underline{R} R} p} & \mathbf{J}_{C_{3 R \underline{R} R} p}
\end{array}\right] \\
& \mathbf{J}_{s_{\underline{\underline{P} R R}} p}=\left[\begin{array}{llllll}
\mathbf{J}_{A_{1 \underline{P} R R} p} & \mathbf{J}_{A_{2 \underline{P} R R} p} & \mathbf{J}_{A_{3 \underline{P} R R} p} & \mathbf{J}_{B_{1 \underline{P} R R} p} & \mathbf{J}_{B_{2 \underline{P} R R} p} & \mathbf{J}_{B_{3 \underline{P} R R} p}
\end{array}\right. \\
& \left.\begin{array}{llllll}
\mathbf{j}_{l_{1 \underline{P} R R} p} & \mathbf{j}_{l_{2 \underline{P} R R} p} & \mathbf{j}_{3 \underline{\underline{P} R R}} & \mathbf{J}_{C_{1 \underline{P} R R}} & \mathbf{J}_{C_{2 \underline{P} R R} p} & \mathbf{J}_{C_{3 \underline{P} R R} p}
\end{array}\right]
\end{aligned}
$$

